\title{
Mechanism Designs for Super Cup Gift Machines and Change Machines
}

\author{
Chien-Yu Lu, ${ }^{1}$ Ching-Chou Tai, ${ }^{1}$ Wen-Yih Horng, ${ }^{1}$ Sun-Chin Huang, ${ }^{1}$ \\ Kai-Yi Hsueh, ${ }^{1}$ Yu-Cheng Liao, ${ }^{2}$ and Te-Jen $\mathrm{Su}^{3,4^{*}}$ \\ ${ }^{1}$ Department of Industrial Education and Technology, National Changhua University of Education, \\ No. 1, Jin-De Road, Changhua City 500207, Taiwan, R. O. C. \\ ${ }^{2}$ Department of Electronic Engineering, The Hong Kong University of Science and Technology, \\ Clear Water Bay, Kowloon 999077, Hong Kong \\ ${ }^{3}$ Department of Electronic Engineering, National Kaohsiung University of Science and Technology, \\ No. 415, Jiangong Rd., Sanmin Dist., Kaohsiung City 807618, Taiwan, R. O. C. \\ ${ }^{4}$ Graduate Institute of Clinical Medicine, Kaohsiung Medical University, \\ No. 100, Shih-Chuan 1st Road, Sanmin Dist., Kaohsiung City 80708, Taiwan, R. O. C.
}

(Received June 1, 2020; accepted January 21, 2021)

Keywords: electronic game machine, slider crank mechanism, hoisting appliance, finite element analysis

The purpose of this research is to explore the mechanism design of gift machines and change machines, develop a new type of electronic game machine, and improve the mechanisms of the old type of change machine. The Super Cup Gift Machine is a newly developed electronic game machine with a novel control mechanism that uses a Fire Electric Pen to negotiate a path, giving players different experiences. The machine's exterior is covered with sheet metal, which not only improves its stability and robustness, but also reduces its cost. The game machine operates using three mechanisms. Firstly, a timing belt pulley and belt driven by a motor driver allows left and right transverse sensors to move in the $X$ direction. Secondly, movement in the $Y$ direction is achieved with a guide bar and a linear bearing with a hoisting appliance. Finally, the reciprocating action in the $Z$ direction is achieved using a slider crank mechanism. The inside of the old type of change machine, which converts coins into tokens, can only store 2000 tokens. To increase the storage capacity to 20000, we first used SOLIDWORKS drawing software to change the existing 3D stereogram; its internal redundant space was put to use, and a coin hopper able to bear the weight of 20000 tokens with a weight sensor was designed. Meanwhile, two inclined planes and an automatic coin-dropping device were added in the coin hopper to prevent multiple coins from dropping at one time and crushing the automatic coin-dropping device. Finally, finite element analysis of the developed structure was performed using ANSYS software, and a physical machine was made by assembling the parts. The designs were tested several times to prove the feasibility of the structure.

*Corresponding author: e-mail: sutj@nkust.edu.tw https://doi.org/10.18494/SAM.2021.2956 


\section{Introduction}

With the changing times, the entertainment provided for modern children is very different from that for children in previous generations. Formerly, children mainly played outdoor games. Nowadays, children play indoor games, and electronic games have become the mainstream. ${ }^{(1)}$ For example, doll machines, in which a claw is manipulated to try and grab a doll from a pile of dolls, used to be popular, and, with the rise of the Internet of Things, ${ }^{(2,3)}$ they have gradually become popular again in recent years owing to improved machines, different playing methods, and diverse prizes. Research has shown that the main reasons for players' interest and persistence in games are the entertainment, pleasure, and curiosity that games provide, while the motivation for seeking entertainment is to experience challenge, achievement, immersion, superiority, and feedback. ${ }^{(4)}$ When people face challenges, the degree of difficulty of that challenge will vary according to people's views and ideas; this is also true in the video game world. Timely adjustment of the difficulty level of a game can keep it challenging and maximize players' sense of achievement, which can increase the freshness and longevity of the game. Therefore, through the development of new game machines and the improvement of old machines, the purpose of our research is to bring more entertainment, pleasure, and excitement to people who love video games. ${ }^{(5)}$

\section{Research Methods and Tools}

We used SOLIDWORKS and ANSYS as research tools. ${ }^{(6,7)}$ Firstly, SOLIDWORKS 3D software was used to draw the individual parts of the mechanism in the proposed design. These drawings were combined, the combined parts were formed, the structures of the combined parts were analyzed with ANSYS, and the results obtained were used to verify the proposed design. SOLIDWORKS software can be easily operated and used to design any mechanism, and the design files can be quickly converted into $2 \mathrm{D}$ engineering drawings. The software also includes design application modules for sheet metal, welding, molding, and curved surfaces to help designers more quickly develop high-quality products. The C package of ANSYS allows designers to quickly solve complex structural engineering problems, provides them with good and clear design decisions, and reduces the cost of physical tests. With the help of the finite element analysis tool in the package, parametric simulation can be carried out to analyze various design schemes, and other physical analysis tools can be easily connected to improve the reliability and accuracy of the results. Common applications of the ANSYS structural analysis package include strength analysis, vibration analysis, fluid dynamics analysis, and collision analysis.

\section{Introduction to Super Cup Gift Machine}

The Super Cup Gift Machine is a video game machine that is easy to operate and suitable for all ages. The game machine uses the game characteristics of a Fire Electric Pen as the design method for the target and obstacle, as shown in Fig. 1. At the beginning of the game, the target 


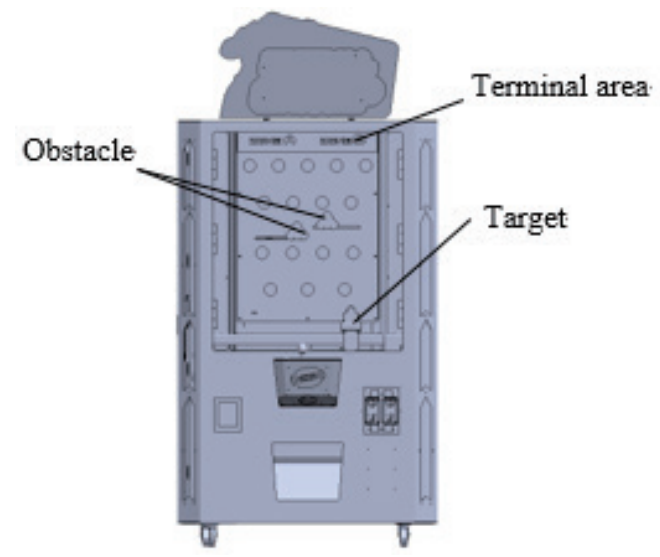

Fig. 1. (Color online) View of Super Cup Gift Machine.

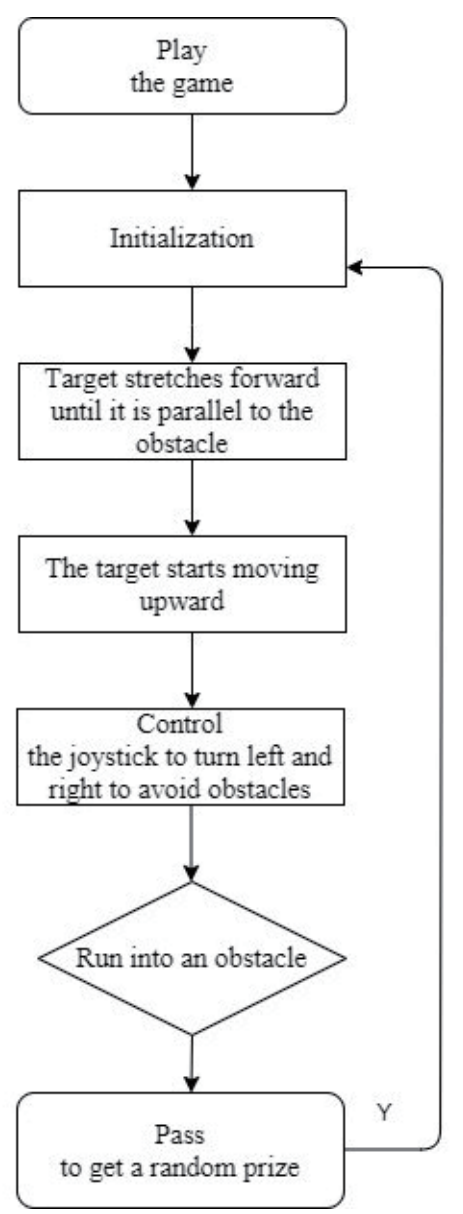

Fig. 2. Flow chart of game on Super Cup Gift Machine.

extends forward until it is parallel to the obstacle, then gradually accelerates as its rises from the bottom of the machine. With the operator controlling the left and right movements of the target to avoid the obstacles using a movement sensor, the target moves forward until it reaches the end point. The player can get random gifts on the left and right sides of the game space when the target collides with the gifts. Conversely, when the target runs into an obstacle, the game will end in failure, as shown in the flow chart of the game in Fig. 2. This section introduces the mechanism and the design concepts, ${ }^{(8)}$ as well as the selection of materials for the overall appearance of the game machine, which is based on the left-handed coordinate system.

The $X$-direction transverse sensor design allows the operator to control the left and right movements of the target using a movement sensor, so that the target can avoid obstacles. A step motor is used to drive the timing belt pulley with positive and negative rotations of the motor. When the belt and the timing belt pulley on both sides mesh and are attached to both sides of the target fixed set, the target can move left and right. The transverse shifting mechanism is fixed on both sides of the $X$ platform by the $X$-axis motor set and the $X$-axis belt holder, as shown in Fig. 3. When designing the $X$-axis motor set, it is necessary to understand the load weight and 


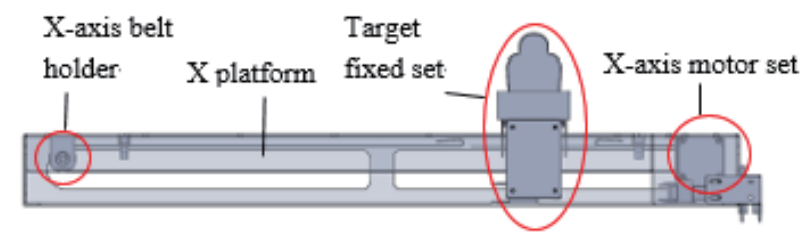

Fig. 3. (Color online) 3D rear view of $X$-direction mechanism.

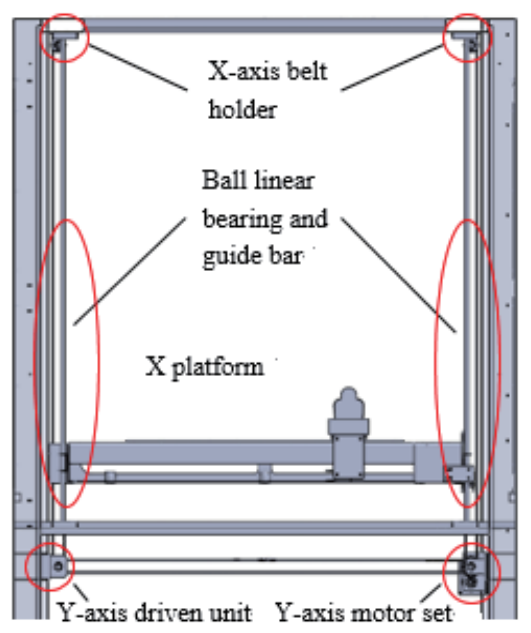

Fig. 4. (Color online) $Y$-direction lifting design.

the space required for the configuration and mechanism action. Firstly, the operating conditions of left and right transverse movements must be set, and an appropriate timing belt pulley and belt, bearing, and self-control axis must be selected according to the operating conditions. The corresponding torsion of the step motor must then be selected according to the weight of the load, and an appropriate coupling must be selected as the connecting device according to the motor axis and the diameter of the self-control axis. The design of the $X$-axis belt holder is the same as that of the belt pulley mechanism in the rear segment of the $X$-axis motor set. This design is a mechanism module, which will be also used in the hoisting mechanism in the $Y$ direction. The $X$-direction transverse design parts include a step motor, a coupling, a flange bearing, a self-control axis, a timing belt pulley, a timing belt, a sliding block, and a slideway. ${ }^{(9-11)}$

The lifting mechanism is designed to make the $X$ platform rise and fall, and its mechanism consists of a $Y$-axis motor set, a $Y$-axis driven unit, a ball linear bearing and guide bar, and an $X$-axis belt holder, as shown in Fig. 4. The combination of the belt pulley and belt is mainly used to match the positive and negative rotations of the step motor. Linear bearings are mounted on both sides of the $X$ platform to enable the platform to move up and down along the guide bar. The $Y$-axis motor set is the driving part of the lifting mechanism, and its parts include a step motor, a coupling, a flange bearing, a timing belt pulley, a timing belt, and 30-tooth and 16-tooth spur gears. The $Y$-axis driving unit is the driving part of the lifting mechanism, which is locked on the U-shaped metal plate holder by the flange bearing, timing belt pulley, and self-control axis. The two belt pulleys of the $Y$-axis motor set are symmetrical, allowing the belt pulley to mesh more precisely with the belt. To make the rotating speed correspond to the amount of movement, when the belt pulley is selected, the same modulus and number of teeth are needed to synchronize the gears. The ball linear bearing and the guide bar are important matching components of the lifting mechanism. The length and diameter of the guide bar are 1112 and $8 \mathrm{~mm}$, respectively, and the guide bar is made from SUJ2 bearing steel. This is a high- 
carbon and high-chromium alloy steel; after tempering and quenching, its hardness is high and uniform, with good performance and high wear resistance and contact fatigue strength. This steel is suitable for manufacturing various mechanical parts, such as bearings, guide pillars, guide bushings, roll shafts, rollers, and balls. The ball linear bearing consists of a round outer cylinder, a steel ball, a steel ball retainer, and dust-proof retaining rings at both ends. The retainer is placed in the outer cylinder and is fixed by the dust-proof retaining rings at both ends, so that the steel ball can repeat the cycle in the track. The body is made of high-quality bearing steel, and the outer cylinder has good hardness after heat treatment to ensure a long lifetime and sliding stability. The steel ball retainer is made of two materials, resin and steel, which can provide different options for noise reduction or high-temperature use. ${ }^{(12,13)}$

The four-bar linkage mechanism is the most common mechanism in the linkage set. "ab" and "ad" are fixed axes, and connecting bar A is a straight line connected by the two fixed axes, also known as the line of centers. Both connecting bar B and connecting bar D may be a joystick or crank, where the joystick is a connecting bar swinging around the fixed shaft, and the crank is a connecting bar rotating $360^{\circ}$ around the fixed shaft. Connecting bar $\mathrm{C}$ is called a connecting bar or a floating bar, which is used to connect the movement sensor or crank, and to transmit motion between them. As shown in Fig. 5, A is a fixed bar and B is a crank. The following are the conditions satisfied by the four-bar linkage mechanism:

$$
\begin{aligned}
& \mathrm{B}+\mathrm{C}+\mathrm{D}>\mathrm{A}, \\
& \mathrm{B}+\mathrm{A}+\mathrm{D}>\mathrm{C} .
\end{aligned}
$$

In the reciprocating block slider crank mechanism, the joystick in the crank joystick mechanism is replaced with a sliding block in the slideway. Stroke $\mathrm{S}$ of this mechanism is twice the length of crank $\mathrm{AB}$, as shown in Fig. 6. The reciprocating motion in the $Z$ direction takes the slider crank mechanism as the design concept, and is installed in the target fixed set, which drives the target forward and backward, as shown in Fig. 7. The main parts of the Z-direction mechanism are a DC reducing motor, a self-control bar, a sliding rail, and a slideway. The selfcontrol crank is $25 \mathrm{~mm}$ long, the floating bar is $70 \mathrm{~mm}$ long, the stroke is $50 \mathrm{~mm}$, twice the

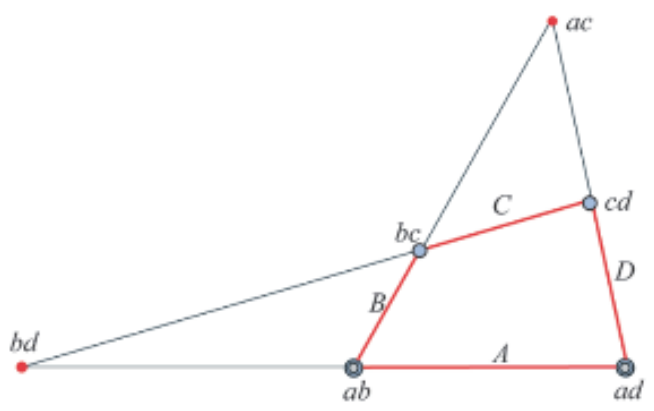

Fig. 5. (Color online) Schematic diagram of the four-bar linkage mechanism. ${ }^{(14)}$ 


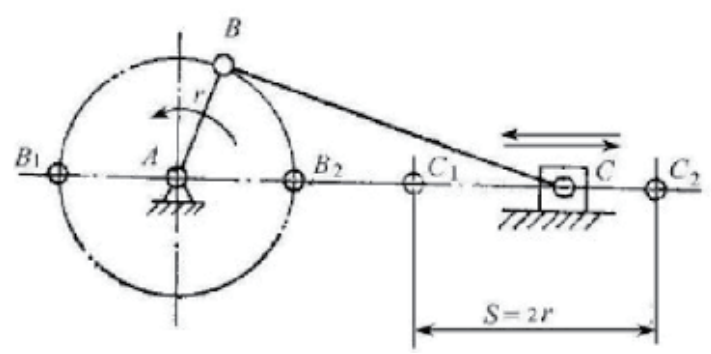

Fig. 6. Schematic diagram of the reciprocating block slider crank mechanism. ${ }^{(15)}$

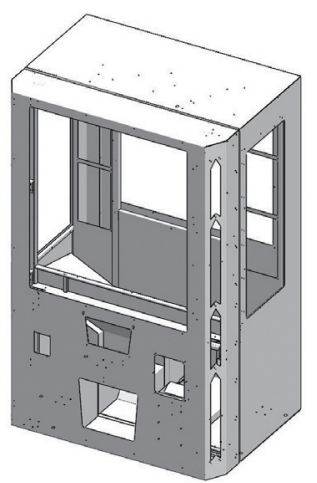

(a)

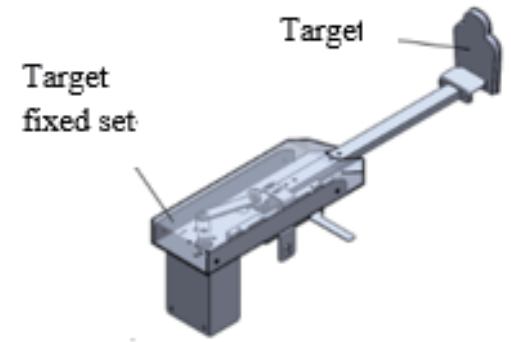

Fig. 7. (Color online) Isometric drawing of Z-direction mechanism.

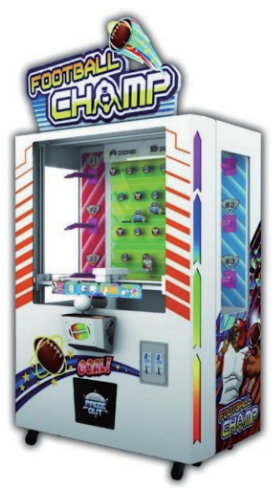

(b)

Fig. 8. (Color online) (a) Equiangular diagram and (b) appearance of Super Cup Gift Machine.

crank's length, and the target only needs to stretch forward $17 \mathrm{~mm}$ to conform to the slider crank mechanism.

Steel can be divided into many specifications, shapes, grades, and surface treatments; each type of steel has different characteristics, resulting in the use of steel in many fields. If good design can be achieved with ordinary carbon steel, then usually a more expensive alloy steel will not be chosen. In this design, the length is $950 \mathrm{~mm}$, the width is $1300 \mathrm{~mm}$, and the height is $1907 \mathrm{~mm}$. Therefore, a cold-rolled steel plate is selected as the material for metal plate machining, as it is not only strong, but it can also meet the designer's price requirement, and is suitable for the appearance of the product. The surface is treated with white varnish, which in addition to its cosmetic function, also prevents the steel plate from rusting. Its machining methods include welding, laser cutting, and boring, as shown in Fig. 8. Common mild steel cold-rolled steel plate is divided into three types: SPCC, SPCD, and SPCE. They are finished steel plate products produced with hot-rolled steel coils as raw materials, using the cold rolling and heat treatment processes. They have a smooth surface, high hardness and strength, and are not easily deformed. ${ }^{(16)}$ After physical assembly of the machine in Fig. 8, the machine was tested many times to determine whether the mechanism design can successfully complete the game flow. 


\section{Introduction to the New Type of Change Machine}

This section describes the internal mechanism of the old type of change machine, which converts coins into tokens, and aspects to be improved. SOLIDWORKS drawing software was used to design the internal mechanism of the new type of change machine, and the structure analysis of its mechanism was conducted to verify its feasibility.

The old type of change machine can only store about 2000 tokens, so we designed a mechanism for a new type of change machine. To increase the number of tokens from 2000 to 20000 , first of all, two refund mechanisms in an old-type change machine were removed, the remaining bill acceptor and coin acceptor were retained, and a new coin hopper mechanism and another newly added coin counter were set up in the space after removal, as shown in Fig. 9. The old and new designs operate differently, using a coin counter and a refund mechanism, respectively. The coin counter in the new design dispenses tokens using a motor to drive a conveyor belt, while the refund mechanism dispenses tokens using a motor to turn a rotating disk. The difference between a coin counter and the refund mechanism is that in the former, coins do not easily get stuck, and the noise made by the conveyance of tokens is also reduced, while coins often get stuck in the refund mechanism in the old type of change machine, and the noise of tokens colliding with metal is also loud. Therefore, it was decided to use this coin counter to address these problems. The coin hopper consists of an automatic coin-dropping device, a weight sensor, an inclined plate, a vibrating motor, and a supporting square tube. Its exterior and the inclined plate were both made of cold-rolled steel plate that was painted black. The exterior of the coin hopper uses the square tube as a framework, ensuring that the entire coin hopper was firmly installed, with no movement.

When the automatic coin-dropping device senses the quantity of tokens in the coin counter, if the quantity is insufficient, the step motor is activated automatically to allow the bucket to rotate $120^{\circ}$ clockwise, so that tokens fall into the coin counter until a sufficient quantity of tokens is sensed. The step motor rotates the bucket counterclockwise to stop dropping coins, as shown in Fig. 10. The motor transmission unit of the automatic coin-dropping device is similar

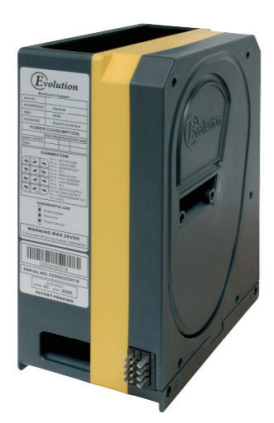

(a)

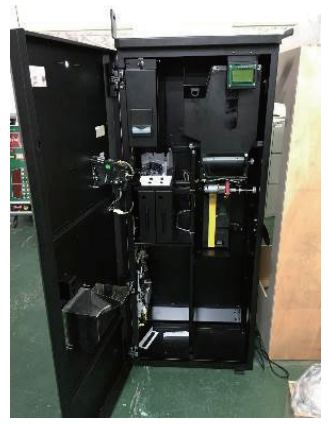

(b)

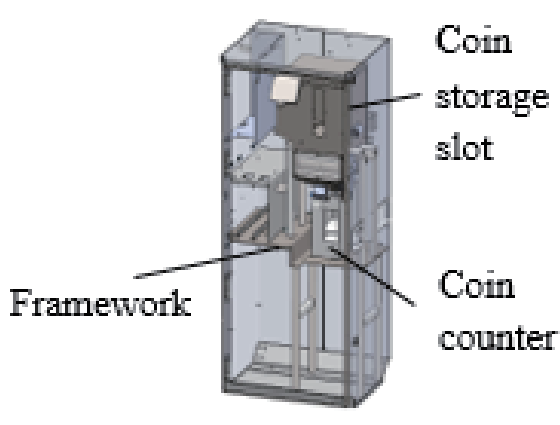

(c)

Fig. 9. (Color online) New type of change machine. ${ }^{(17)}$ (a) Coin Hopper. (b) Mechanism of new type of change machine. (c) 3D sketch of coin hopper coin counter. 


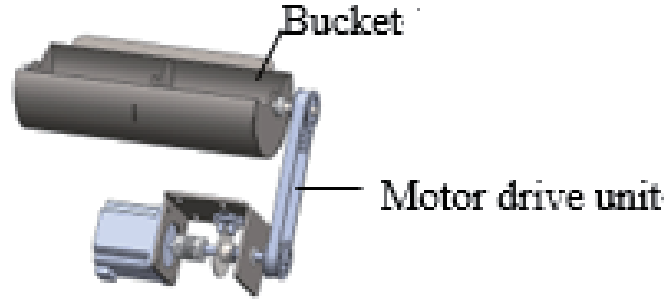

Fig. 10. (Color online) Mechanism of automatic coin-dropping device.

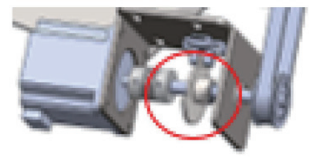

(a)

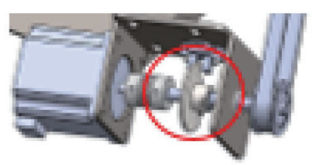

(b)

Fig. 11. (Color online) Schematic diagram of lightshading sheet induction set. (a) Not started. (b) Bucket rotates $120^{\circ}$ clockwise.

to that of the $X$-direction transverse shifting mechanism design mentioned in Sect. 3. The lightshading sheet induction set used in the new type of change machine is composed of an L-type NPN output groove connector-type photo interrupter and a self-made light-shading sheet. This is to ensure that the induction bucket can rotate $120^{\circ}$ clockwise without deviation, as shown in Fig. 11.

The vibrating motor of the coin-dropping device is a DC brush motor. Its strength depends on the size and speed of the pendulum. The vibrating motor is secured below a slanting baffle. After the external dimensions of the motor are measured and drawn using SOLIDWORKS, the motor support is made by 3D line printing. To prevent the vibrating motor from making noise when it touches the slanting baffle, a buffer layer is pasted on the outside of the motor to prevent excessive noise. The vibrating motor is used to prevent the generation of compressive stress when coins overlap, and to prevent coins from becoming stuck; the vibration force is transmitted to the whole steel plate so that tokens drop smoothly. ${ }^{(18)}$

General square tubes, which have excellent machinability and plasticity, are widely used in buildings, bridges, and factory buildings, and can also improve the use of space. Square tubes can easily be used with other building materials. Square tubes have high vibration resistance and high bearing capacity. The square tubes designed in this study to support the coin-dropping device have dimensions of $28 \times 28 \times 2 \mathrm{~mm}^{3}$, and the total length of tubes used is $347.8 \mathrm{~mm}$. The square tube areas used for support assist the slanting baffles in bearing the weight of tokens. Because one token is about $8.5 \mathrm{~g}$, assuming that 10000 tokens are poured into the coin hopper, the two slanting baffles must bear a weight of more than $85 \mathrm{~kg}$ without breaking under sustained pressure. Moreover, it is necessary to place the entire coin hopper on the framework, so that the coin hopper can be smoothly placed inside the change machine, as shown in Fig. 12. The framework is composed of a $Z$-type metal plate and several rectangular tubes, all $40 \times 20 \times 2 \mathrm{~mm}^{3}$. Various bevels are cut out using laser cutting, and the frame structure is welded onto the $Z$-type metal plate, forming an important supporting structure for the whole coin hopper.

In the experimental evaluation, ANSYS software was used for structural analysis and overall testing. We examined whether the force borne by the two slanting baffles and supporting square 


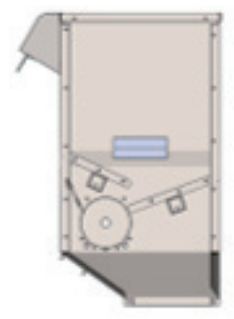

(a)

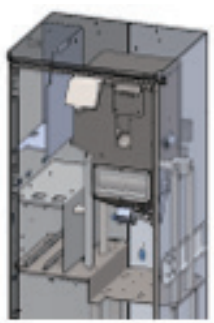

(b)

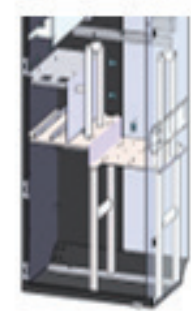

(c)

Fig. 12. (Color online) Schematic diagram of supporting square tubes and 3D view of the framework. (a) Supporting two slanting baffles. (b) Coin hopper placed on the framework. (c) Framework 3D drawing.

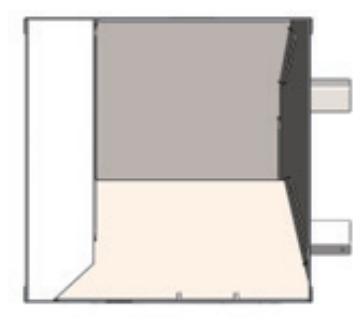

(a)

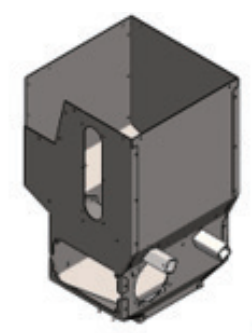

(b)

Fig. 13. (Color online) Original 3D view of the coin hopper. (a) Top view of coin hopper. (b) Isometric drawing of coin hopper.

tubes was likely to result in structural failure when a large number of tokens were poured into the coin hopper, and whether the structure would seriously deform under long-term heavy loads.

We next discuss the stress and strain generated by the weight of the tokens, as well as the total deformation between the coin hopper and the framework, where the parts not affected by the force were removed, as shown in Fig. 13. By setting the downward force on the two slanting baffles in the coin hopper to $42.5 \mathrm{~kg}$ each, we found that if the slanting baffles are subject to a weight of more than $42.5 \mathrm{~kg}$ for a long time, they are likely to deform or fracture at the welds. In another structural analysis, 10000 tokens were poured into the coin hopper, so that the weight reached more than $100 \mathrm{~kg}$, and the hopper was assumed to be installed on the framework, as shown in Fig. 12(b). The analysis proved that the supporting beams on both sides had significantly different deformation degrees, where the serious deformation of one side was caused by the lack of support against the external framework, and that the upper and lower beams were not aligned.

\section{Conclusions}

The mechanisms in this study were all designed in similar ways. The design of the mechanisms was realized by human thinking and creative ideas. Different mechanism designs can be created by following basic design principles from ancient to modern times and by using different methods. We conducted a structure analysis and action simulation of the mechanisms of the Super Cup Gift Machine and a new type of change machine, and put forward conclusions 
and suggestions for future work based on the test results of the physical machines. The Super Cup Gift Machine is mainly divided into mechanisms operating in three directions, where the $X$ - and $Y$-direction mechanisms have step motors to drive timing belt pulleys and belts for transmission. After repeated tests of several physical machines, it was found that the guide bar tended to generate excessive vibration during the hoisting process of the hoisting appliance, which may shorten the service life of the product and reduce its durability. In contrast, the shifting mechanism of the $X$-direction transverse sensor was very smooth, and the target of the left and right was synchronized with the controller's control. The $Z$-direction slider crank mechanism also had no dead-point problem and could complete the reciprocating action smoothly. In the test involving a large number of tokens being fed into the new type of change machine, compared with the old type of change machine, the number of stuck coins was significantly reduced. Because of the increased weight of the tokens, more than 20000 tokens could be fed into the coin hopper, exceeding the original capacity of 2000 tokens. However, the results of the structural analysis show that when 10000 tokens are fed into the hopper, the two slanting baffles and the framework structure inside the coin hopper suffer deformation. Therefore, future work will explore whether the slanting baffles and the framework should be removed, and look at alternative ways to install the coin hopper in the change machine to increase the token capacity.

\section{References}

1 C. Y. Tsai: Development and Transformation of Children's Games in Taiwan: Through the Discussion of Childhood Games from Different Generations, Master's Thesis (Institute of Education, National Chiayi University, Taiwan, R. O. C., 2010)

2 L. Atzori, A. Iera, and G. Morabito: Comput. Netw. 54 (2010) 2787. https://dori.org/10.1016/j.comnet.2010.05.010

3 D. Miorandi, S. Sicari, F. De Pellegrini, and I. Chlamtac: Ad Hoc Netw. 10 (2012) 1497. https://doi.org/10.1016/ j.adhoc. 2012.02.016

4 Y. T. Ma: Relevance of Computer Game Characteristics to Gamers' Psychological Feelings - Taking College Students Group as an Example, Master's Thesis (College of Design, Ming Dao University, Taiwan R. O. C., 2010)

5 T. W. Malone and M. R. Lepper: Learn. Instr. 3 (1987) 223. https://learningenvironmentsdesign.pressbooks. com/chapter/malone-lepper-making-learning-fun-a-taxonomy-of-intrinsic-motivations-for-learning/

6 SOLIDWORKS 3D Design: http://www.swtc.com/cht/index.php (accessed June 2018).

7 Structural Analysis: https://www.ansys.com/ (accessed June 2018).

8 C. K. Lee, J. Lee, P. W. Lo, H. L. Tang, W. H. Hsiao, J. Y. Liu, and T. L. Lin: AUMS 1 (2011) 93. https://doi. org/10.5875/ausmt.vli1.74

9 TECO Precision Electric Co., Ltd.: Stepping Motor-DST56EL62A, http://www.tedmotors.com/tw/pro/detail. php?pid=170\&cid=76\&f=76 (accessed June 2018).

10 Xiang Yun Trading Co., Ltd.: Coupling (MFS), http://mlmf.com/ (accessed June 2018).

11 Donglong Co., Ltd.: T5 Standard timing pulley, http://edpt.com.tw/big5/index.php (accessed June 2018).

12 Sheng metallocene Co., Ltd.: SUJ2 Bearing steel, http:/www.sheng-maw.com.tw/productshow.php?id=246 (accessed June 2018).

13 Telida Trading Co., Ltd.: Ball Linear Bearing Lengthened LM-LUU, http://www.cyrus-linear.com/productdetail.php?id=16\&lang=tw (accessed June 2018).

14 Y. L. Ke, and J. A. Pan: Mechanism Principle II (Taike Co., Ltd. Taipei, 2011).

15 D. Zhao: 2nd Int. Conf. Education Technology, Management and Humanities Science (ETMHS 2016) 87-92.

16 China Steel Corporation: Cold rolled products, http://www.csc.com.tw/index.html (accessed June 2018).

17 Feiloli Electronics Co., Ltd.: Currency exchange machine, http://www.feiloli.com.tw/ (accessed June 2018).

18 Qing-Chang Wu: High Bearing Capacity of Far East Machinery Large-size Rectangular Tubes to Push Up the Floor Height Upward, Economic Daily News, United Daily News Group (July 2013), http://edn.udn.com/news/ view.jsp?aid=642661\&cid=7\# (accessed June 2018). 